\title{
Endoscope-assisted transoral approach to parapharyngeal space tumor: a case report
}

\begin{abstract}
Introduction: Parapharyngeal space tumors account for $0.5 \%$ of Head and neck tumors. Various surgical approaches have been described for the management of these tumours. This study compares endoscope-assisted transoral approach with the traditional approaches.

Objective: To present an endoscopic, transoral approach for the treatment of a benign prestyloid PPS tumor.

Study design: Case report and review of the literature.

Patient: A 35year old female came for evaluation of right tonsillar fossa swelling of 1year duration. The CT showed a mixed density nodular lesion occupying right parapharyngeal space.
\end{abstract}

Surgical intervention: Endoscope-assisted transoral approach for removal of PPS tumor.

Results: The patient was discharged on postoperative day 1 in stable condition. There have been no complications with 2years of follow-up.

Conclusion: Endoscopic assisted transoral approach should be one of the primary surgical options for benign tumor not involving the critical structures in PPS.

Keywords: parapharyngeal space tumors, endoscope, transoral approach
Volume 2 Issue 3 - 2015

\author{
Sanjay Helale, Preety Ninan, Abhishek Kumar \\ Ramadhin \\ Department of Otolaryngology, Kohinoor Superspeciality \\ Hospital, India
}

\begin{abstract}
Correspondence: Sanjay Helale, Department of
Otolaryngology, Kohinoor City Kirol Road, Off LBS Road, Kurla (West) Mumbai 400070, India, Email drsanjayhelale@gmail.com
\end{abstract}

Received: March 03, 2015 | Published: April 09, 2015
Abbreviations: PPS, parapharyngeal space; CT, computed tomography; MRI, magnetic resonance imaging

\section{Introduction}

Parapharyngeal space tumors are extremely rare and it is about $0.5 \%$ of Head and neck tumor. ${ }^{1}$ Surgery is often challenging due to limited surgical exposure and morbidity associated with them. There are various ways to reach the parapharyngeal space masses; transoral and external approaches (i.e. transcervical, transparotid, transmandibular and combined approach). The choice of surgical approach depends on its location i.e. prestyloid or post styloid, on the size of the tumor, vascularity of tumor, its relationship to the great vessels, and suspicion of malignancy. ${ }^{2,3}$

Transoral approach was first described by Ehrlich in 1950 and it was indicated for small, non vascular tumors, as it offers poor exposition and does not give adequate control in the event of haemorrhage. ${ }^{4}$ But with advent of endoscope assistance to this transoral approach, it provides direct and magnified visualization, less amount of bleeding, reduced tissue damage, improved cosmetic appearance, and fewer wound-related complications and less post-operative morbidity.

\section{Case report}

A 35year old female was referred for evaluation of right tonsillar fossa swelling of 1year duration with more noticeable increase in size in last 2 months. The CT was ordered and showing mixed density nodular lesion occupying right parapharyngeal space. The patient denied symptoms such as shortness of breath, apnea, dysphasia, and voice changes or globus sensation. No symptoms or signs were suggestive of nerve involvement such as paresis, facial twitching, ear pain and odynophagia.
On examination, there was fullness in right peritonsillar fossa, leading to bulge of adjacent, tonsil, soft palate, and uvula because of mass effect. There was no pain or tenderness on palpation or by movement of neck. Hematological and biochemical investigations were all within normal limits. CT scan showed an encapsulated, well marginated, smooth-bordered, mixed density mass in the right parapharyngeal space, and there was no involvement of any vascular or nervous structures. The tumor was $4.0 \times 4.0 \times 5.0 \mathrm{~cm}$ and situated in the parapharyngeal space posterior to pterygoid plates and anterior to styloid process (Figure 1A\&B).
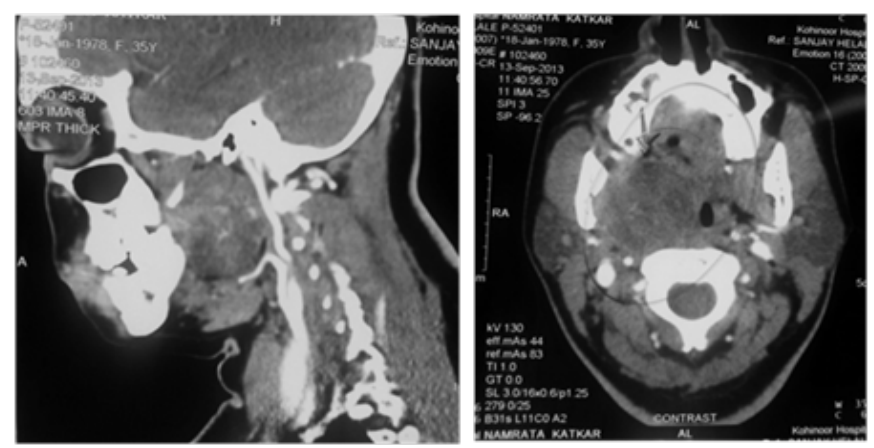

Figure I (A) CT scan with Contrast (Coronal section) - Well defined mildly enhancing Parapharyngeal lesion. (B) CT scan with Contrast- Axial section Parapharyngeal lesion not involving the great vessels and parotid gland.

\section{Surgical technique}

After proper exposure of the oral cavity by using a Boyle-Davis gag, a transoral pharyngotomy was performed by linear-vertical incision through the overlying mucosa, buccopharyngeal fascia and pharyngeal muscle. Dissection had been carried out down to the 
level of the tumor. It was performed with assistance of 0 and 30 rigid endoscopes. A combination of sharp and blunt dissection was used to expose and completely free the tumor from surrounding structures. Blunt dissection was done on capsule (endoscope assisted) and care was taken not to rupture it. There was no damage to surrounding nervous structures or vasculature. The tumor was dissected en bloc under direct endoscopic visualization (Figure 2). After ensuring there was no residual tumor, the pharyngotomy was repaired in layers. The patient was discharged home on the next day of surgery with total blood loss less than $20 \mathrm{ml}$.

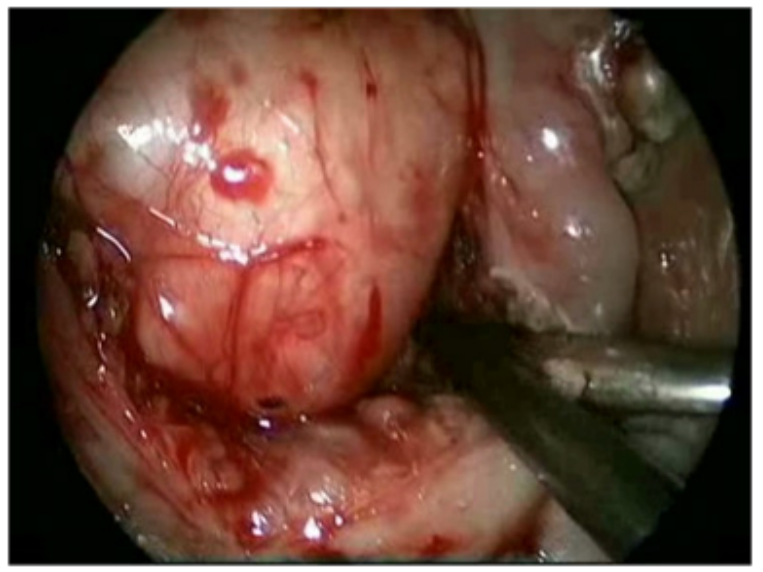

Figure 2 Endoscopic picture illustrating the parapharyngeal tumor during dissection.

The gross specimen was $6 \times 4 \times 5 \mathrm{~cm}^{3}$ in dimensions and had characteristics of well-demarcated, encapsulated, and uniformly soft with smooth edges (Figure 3) and confirmed on histopathology as pleomorphic adenoma (Figure 4). Since the surgery the patient has been asymptomatic. The follow-up examinations did not reveal any signs of complication or recurrence in 2 years of follow up and still we are following up the patient.

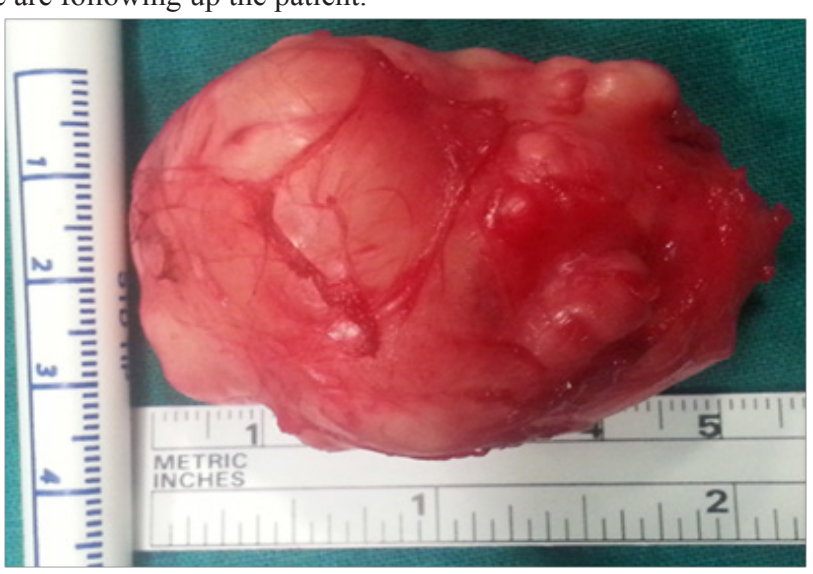

Figure 3 Gross specimen showing the parapharyngeal tumor.

\section{Discussion}

Because of complex anatomy and pathology, the appropriate surgical management differs widely. The different surgical approaches to the parapharyngeal space are external approaches and transoral. Each approach has its advantages and pitfalls.

Because of its location deep within the neck, the space is difficult to examine by ordinary methods, but can be well demonstrated in the axial section by computed tomography and MRI, ${ }^{5}$ based on that the approach can be decided.

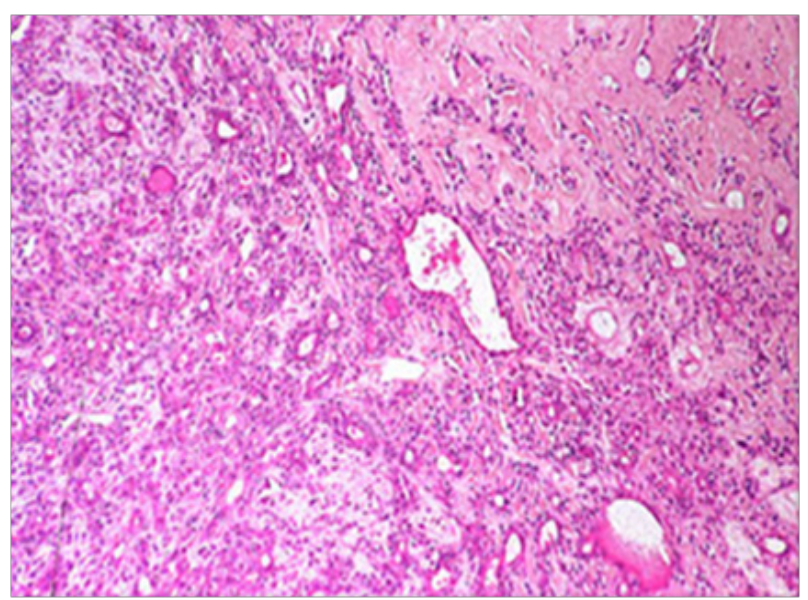

Figure 4 Histological features suggestive of pleomorphic adenoma.

Transoral approach was first described by Ehrlich in 1950 and it was indicated for small, non-vascular tumors. ${ }^{4}$ In 1963, McElroth et al described the use of this approach in a study of 112 patients along with ligature of external carotid artery. ${ }^{6}$ In 1988 Goodwin and Chandler advocated this approach to give adequate access to the parapharyngeal space. In their study of six cases, there were no surgical complications and blood loss was minimal. ${ }^{7}$

This approach is essentially the same as an extended tonsillectomy. Using $0^{0}$ and angled endoscopes, provided excellent visualization of areas beyond line of sight and enabled confirmation of adequate hemostasis and complete resection prior to closure, which may potentially decrease the recurrence rate. The improved visualization provided by the endoscope allowed us to take advantage of the low morbidity.

Biggest disadvantage is that the neck is not open and the vessels not exposed in the event of injury to the major vessels. Hence this approach requires appropriate patient selection. We believe that the transoral approach is not advised for malignancies, for tumors invading the bony skull base or invading intracranially, for vascular tumors, or for tumors surrounding the facial nerve (such as a dumbbell tumor). In these cases, an open approach may be better suited.

In cases that do not require extensive access, however, the transoral approach can offer the least amount of trauma, minimal post-op morbidities and immediate return of function. ${ }^{2}$ Hence, lessen the relative scar burden, spare the patient the risks of facial nerve dissection, and preserving the greater auricular nerve, which could get damaged in traditional transcervical and trans parotid approach.

Every case of parapharyngeal tumor should require distinct approach, careful evaluation of risk and benefit ratio of individual case. When there is suspicion of malignancy, involvement of neurovascular structures or reasonably large tumor, then external approach for removal of complete tumor is a necessity. Large tumors that extend into the skull base may require a transcervical transmandibular approach. ${ }^{8}$ But when tumor is benign, small, and no complicating factors transoral endoscopic assisted approach would be more appropriate. 


\section{Conclusion}

Traditionally surgeons were considering, transoral rout to the parapharyngeal space as poor and unsafe. But with advent of videoassisted endoscope assistance, transoral route provides direct and magnified visualization. This is allowing excision of sizable benign tumors near to critical anatomical structures. Endoscopic assisted transoral approach should be one of the primary surgical options for benign tumor not involving the critical structures.

\section{Acknowledgments}

None.

\section{Conflicts of interest}

The authors declare that there are no conflicts of interest.

\section{Funding}

None.

\section{References}

1. Khafif A, Segev Y, Kaplan DM, et al. Surgical management of parapharyngeal space tumors: a 10-year review. Otolaryngol Head Neck Surg. 2005;132(3):401-406.

2. Cohen SM, Burke BB, Netterville JL. Surgical Management of Parapharyngeal Space Masses. Head Neck. 2005;27(8):669-675.
3. Bozza F, Vigili MG, Ruscito P, et al. Surgical management of parapharyngeal space tumours: results of 10-year follow-up. Acta Otorhinolaryngol Ital. 2009;29(1):10-15.

4. Ehrlich H. Mixed tumours of the pterygomaxillary space; operative removal; oral approach. Oral Surg Oral Med Oral Pathol. 1950;3(11):1366-1371.

5. Maheshwar AA, Kim EY, Pensak ML, et al. Roof of the parapharyngeal space: defining its boundaries and clinical implications. Ann Otol Rhinol Laryngol. 2004;113(4):283-288.

6. Goodwin WJ Jr, Chandler JR. Transoral excision of lateral parapharyngeal space tumors presenting intraorally. Laryngoscope. 1988;98(3):266-269.

7. Unger JM, Chintapalli KN. Computed tomography of the parapharyngeal space. J Comput Assist Tomogr. 1983;7(4):605-609.

8. Iseri M, Ozturk M, Kara A, et al. Endoscope-assisted transoral approach to parapharyngeal space tumors. Head Neck. 2015;37(2): 243-248.

9. Mcelroth DC, Remine WH, Devine KD. Tumours of parapharyngeal region. Surgery Gynaecol Obstet. 1963;116:88-96.

10. Chen WL, Wang YY, Zhang DM, et al. Endoscopy-assisted transoral resection of large benign parapharyngeal space tumors. $\mathrm{Br} J$ Oral Maxillofac Surg. 2014;52(10):970-973.

11. Li SY, Hsu CH, Chen MK. Minimally invasive endoscope-assisted transoral excision of huge parapharyngeal space tumors. Auris Nasus Larynx. 2015;42(2):179-182. 